Bull. Korean Math. Soc. 50 (2013), No. 4, pp. 1315-1328

http://dx.doi.org/10.4134/BKMS.2013.50.4.1315

\title{
ON SOME MODULAR EQUATIONS OF DEGREE 5 AND THEIR APPLICATIONS
}

\author{
Dae Hyun Paek and Jinhee Yi
}

\begin{abstract}
We first derive several modular equations of degree 5 and present their concise proofs based on algebraic computations. We then establish explicit relations and formulas for some parameterizations for the theta functions $\varphi$ and $\psi$ by using the derived modular equations. In addition, we find specific values of the parameterizations and evaluate some numerical values of the Rogers-Ramanujan continued fraction.
\end{abstract}

\section{Introduction}

We begin this section by introducing Ramanujan's definition of his general theta function. For $|a b|<1$, define

$$
f(a, b):=\sum_{n=-\infty}^{\infty} a^{n(n+1) / 2} b^{n(n-1) / 2} .
$$

Note that two special cases of $f(a, b)$ are defined by, for $|q|<1$,

$$
\varphi(q):=f(q, q)=\sum_{n=-\infty}^{\infty} q^{n^{2}}=\left(-q ; q^{2}\right)_{\infty}^{2}\left(q^{2} ; q^{2}\right)_{\infty}
$$

and

$$
\psi(q):=f\left(q, q^{3}\right)=\sum_{n=0}^{\infty} q^{n(n+1) / 2}=\frac{\left(q^{2} ; q^{2}\right)_{\infty}}{\left(q ; q^{2}\right)_{\infty}}
$$

where

$$
(a ; q)_{\infty}:=\prod_{n=0}^{\infty}\left(1-a q^{n}\right)
$$

Received September 27, 2012; Revised November 15, 2012.

2010 Mathematics Subject Classification. Primary 11F27, 33C90; Secondary 11F20, $33 \mathrm{C} 05,33 \mathrm{C} 75$.

Key words and phrases. theta functions, modular equations, continued fractions.

This work was supported by the Education Research Institute, Busan National University of Education in 2013. 
Let $a, b$, and $c$ be arbitrary complex numbers except that $c$ cannot be a nonpositive integer. Then, for $|z|<1$, the Gaussian or ordinary hypergeometric function ${ }_{2} F_{1}(a, b ; c ; z)$ is defined by

$$
{ }_{2} F_{1}(a, b ; c ; z):=\sum_{n=0}^{\infty} \frac{(a)_{n}(b)_{n}}{(c)_{n} n !} z^{n},
$$

where $(a)_{0}:=1$ and $(a)_{n}:=a(a+1)(a+2) \cdots(a+n-1)$ for each positive integer $n$.

Now the complete elliptic integral of the first kind $K(k)$ is defined by

$$
K(k):=\int_{0}^{\pi / 2} \frac{d \theta}{\sqrt{1-k^{2} \sin ^{2} \theta}}=\frac{\pi}{2}{ }_{2} F_{1}\left(\frac{1}{2}, \frac{1}{2} ; 1 ; k^{2}\right)=\frac{\pi}{2} \varphi^{2}\left(e^{-\pi \frac{K^{\prime}}{K}}\right),
$$

where $0<k<1, K^{\prime}=K\left(k^{\prime}\right)$, and $k^{\prime}=\sqrt{1-k^{2}}$. The number $k$ is called the modulus of $K$ and $k^{\prime}$ is called the complementary modulus.

Let $K, K^{\prime}, L$, and $L^{\prime}$ denote complete elliptic integrals of the first kind associated with the moduli $k, k^{\prime}, l$, and $l^{\prime}$, respectively, where $0<k<1$ and $0<l<1$. Suppose that

$$
\frac{L^{\prime}}{L}=n \frac{K^{\prime}}{K}
$$

holds for some positive integer $n$. A relation between $k$ and $l$ induced by (1.2) is called a modular equation of degree $n$.

If we set

$$
q=\exp \left(-\pi \frac{K^{\prime}}{K}\right) \text { and } q^{\prime}=\exp \left(-\pi \frac{L^{\prime}}{L}\right),
$$

we see that (1.2) is equivalent to the relation $q^{n}=q^{\prime}$. Thus, a modular equation can be viewed as an identity involving theta functions at the arguments $q$ and $q^{n}$.

Note that the definition of a modular equation mentioned above is the one used by Ramanujan, but we emphasize that there are several definitions of a modular equation in the literature. For example, refer the books by R. A. Rankin in [7] and B. Schoeneberg in [8] for other definitions of a modular equation. Following Ramanujan, set $\alpha=k^{2}$ and $\beta=l^{2}$, then we say that $\beta$ has degree $n$ over $\alpha$. By the relationship between complete elliptic integrals of the first kind and hypergeometric function, we have

$$
n \frac{{ }_{2} F_{1}\left(\frac{1}{2}, \frac{1}{2} ; 1 ; 1-\alpha\right)}{{ }_{2} F_{1}\left(\frac{1}{2}, \frac{1}{2} ; 1 ; \alpha\right)}=\frac{{ }_{2} F_{1}\left(\frac{1}{2}, \frac{1}{2} ; 1 ; 1-\beta\right)}{{ }_{2} F_{1}\left(\frac{1}{2}, \frac{1}{2} ; 1 ; \beta\right)} .
$$

Let $z_{n}=\varphi^{2}\left(q^{n}\right)$. Then the multiplier $m$ for degree $n$ is defined by

$$
m:=\frac{\varphi^{2}(q)}{\varphi^{2}\left(q^{n}\right)}=\frac{z_{1}}{z_{n}} .
$$

Next we introduce the definitions of 4 parameterizations for the theta functions $\varphi$ and $\psi$ from $[9,11,13]$. For any positive real numbers $k$ and $n$, define 
$h_{k, n}$ by

$$
h_{k, n}:=\frac{\varphi(q)}{k^{1 / 4} \varphi\left(q^{k}\right)},
$$

where $q=e^{-\pi \sqrt{n / k}}$, define $h_{k, n}^{\prime}$ by

$$
h_{k, n}^{\prime}:=\frac{\varphi(-q)}{k^{1 / 4} \varphi\left(-q^{k}\right)},
$$

where $q=e^{-2 \pi \sqrt{n / k}}$, define $l_{k, n}$ by

$$
l_{k, n}:=\frac{\psi(-q)}{k^{1 / 4} q^{(k-1) / 8} \psi\left(-q^{k}\right)},
$$

where $q=e^{-\pi \sqrt{n / k}}$, define $l_{k, n}^{\prime}$ by

$$
l_{k, n}^{\prime}:=\frac{\psi(q)}{k^{1 / 4} q^{(k-1) / 8} \psi\left(q^{k}\right)},
$$

where $q=e^{-\pi \sqrt{n / k}}$

Moreover, in [9, 11, 13], several new modular equations for the theta functions were derived, some explicit relations and formulas for the parameterizations were offered, and some values of the parameterizations were determined. Whereas, in [6, 12], some modular equations of degrees 3 and 9 for the theta functions $\varphi$ and $\psi$ were derived in order to establish explicit relations and formulas for the parameterizations for $h_{k, n}, h_{k, n}^{\prime}, l_{k, n}$, and $l_{k, n}^{\prime}$ and show some applications of the modular equations to evaluations of the cubic continued fraction and the theta function $\psi$.

In this paper, we derive some modular equations of degree 5 for the theta functions $\varphi$ and $\psi$ and present their concise proofs based on algebraic computations as in $[6,12]$. We also find explicit relations and formulas for the corresponding parameterizations, and evaluate some numerical values of $h_{5, n}$, $h_{5, n}^{\prime}, l_{5, n}$, and $l_{5, n}^{\prime}$ by employing the relations and formulas established earlier. Moreover, we use these values to evaluate some specific values of the RogersRamanujan continued fraction.

\section{Preliminary results}

In this section, we introduce basic theta function identities that will play key roles in deriving some modular equations. Let $k$ be the modulus as in (1.1). Set $x=k^{2}$ and also set

$$
k^{2}=x=1-\frac{\varphi^{4}(-q)}{\varphi^{4}(q)} .
$$

Then

$$
\varphi^{2}(q)={ }_{2} F_{1}\left(\frac{1}{2}, \frac{1}{2} ; 1 ; x\right)=: z,
$$


where

$$
q=e^{-y}:=\exp \left(-\pi \frac{{ }_{2} F_{1}\left(\frac{1}{2}, \frac{1}{2} ; 1 ; 1-x\right)}{{ }_{2} F_{1}\left(\frac{1}{2}, \frac{1}{2} ; 1 ; x\right)}\right)=\exp \left(-\pi \frac{K\left(k^{\prime}\right)}{K(k)}\right) .
$$

Lemma 2.1 ([1], Theorem 5.4.1). If $x$, $q$, and $z$ are related by (2.1), (2.2), and (2.3), then
(i) $\varphi(q)=\sqrt{z}$,
(ii) $\varphi(-q)=\sqrt{z}(1-x)^{1 / 4}$,
(iii) $\varphi\left(-q^{2}\right)=\sqrt{z}(1-x)^{1 / 8}$.

Lemma 2.2 ([1], Theorem 5.4.2). If $x, q$, and $z$ are related by (2.1), (2.2), and (2.3), then

(i) $\psi(q)=\sqrt{\frac{1}{2} z}\left(\frac{x}{q}\right)^{1 / 8}$,

(ii) $\psi(-q)=\sqrt{\frac{1}{2} z}\left(\frac{x(1-x)}{q}\right)^{1 / 8}$,

(iii) $\psi\left(q^{2}\right)=\frac{1}{2} \sqrt{z}\left(\frac{x}{q}\right)^{1 / 4}$.

Lemma 2.3 ([2], Entry 13, Chapter 19). Let $\beta$ be the fifth degree and $m=\frac{z_{1}}{z_{5}}$. Then

(i) $\left(\frac{\beta}{\alpha}\right)^{1 / 4}+\left(\frac{1-\beta}{1-\alpha}\right)^{1 / 4}-\left(\frac{\beta(1-\beta)}{\alpha(1-\alpha)}\right)^{1 / 4}=m$,

(ii) $\left(\frac{\alpha}{\beta}\right)^{1 / 4}+\left(\frac{1-\alpha}{1-\beta}\right)^{1 / 4}-\left(\frac{\alpha(1-\alpha)}{\beta(1-\beta)}\right)^{1 / 4}=\frac{5}{m}$.

Lemma 2.4 ([11], Theorem 2.2). For any positive real number $k$,

$$
h_{k, 1}=1 \text {. }
$$

Lemma 2.5 ([13], Theorem 2.3). For any positive real number $k$,

$$
l_{k, 1}=1 \text {. }
$$

We recall that the Rogers-Ramanujan continued fraction $R(q)$ is defined by, for $|q|<1$,

$$
R(q):=\frac{q^{1 / 5}}{1}+\frac{q}{1}+\frac{q^{2}}{1}+\frac{q^{3}}{1}+\ldots=q^{1 / 5} \frac{f\left(-q,-q^{4}\right)}{f\left(-q^{2},-q^{3}\right)} .
$$

We also define

$$
S(q):=-R(-q)
$$

The following results show general formulas for $R\left(e^{-2 \pi \sqrt{n / 5}}\right)$ and $S\left(e^{-\pi \sqrt{n / 5}}\right)$ in terms of $h_{k, n}$ and $l_{k, n}$.

Lemma 2.6 ([13], Theorem 6.1). For any real number $n$, we have 
(i) $\frac{1}{R^{5}\left(e^{-2 \pi \sqrt{n / 5}}\right)}-11-R^{5}\left(e^{-2 \pi \sqrt{n / 5}}\right)=5 \sqrt{5} h_{5, n}^{2} l_{5, n}^{4}$,
(ii) $\frac{1}{S^{5}\left(e^{-\pi \sqrt{n / 5}}\right)}+11-S^{5}\left(e^{-\pi \sqrt{n / 5}}\right)=5 \sqrt{5} h_{5, n}^{4} l_{5, n}^{2}$.

\section{Modular equations}

In [3], there are Ramanujan's 23 eta function identities, which are certain types of modular equations. In this section, we derive some modular equations of degree 5 and present their proofs based on concise algebraic computations by employing the theory of theta functions in the spirit of Ramanujan. In addition, we establish some explicit relations and formulas for $h_{5, n}, h_{5, n}^{\prime}, l_{5, n}$, and $l_{5, n}^{\prime}$ by employing these modular equations.

Theorem 3.1. If $P=\frac{\varphi(q)}{\varphi\left(q^{5}\right)}$ and $Q=\frac{\varphi(-q)}{\varphi\left(-q^{5}\right)}$, then

$$
\frac{P}{Q}+\frac{Q}{P}+4=P Q+\frac{5}{P Q} \text {. }
$$

Proof. By Lemma 2.1(i) and (ii),

$$
P=\sqrt{\frac{z_{1}}{z_{5}}} \text { and } Q=\sqrt{\frac{z_{1}}{z_{5}}}\left(\frac{1-\alpha}{1-\beta}\right)^{1 / 4},
$$

where $\beta$ has degree 5 over $\alpha$. Thus

$$
\frac{Q}{P}=\left(\frac{1-\alpha}{1-\beta}\right)^{1 / 4}
$$

By Lemma 2.3,

$$
\left(\frac{\beta}{\alpha}\right)^{1 / 4}+\frac{P}{Q}-\left(\frac{\beta}{\alpha}\right)^{1 / 4} \frac{P}{Q}=P^{2}
$$

and

$$
\left(\frac{\alpha}{\beta}\right)^{1 / 4}+\frac{Q}{P}-\left(\frac{\alpha}{\beta}\right)^{1 / 4} \frac{Q}{P}=\frac{5}{P^{2}} .
$$

Combining and rewriting above two equations in terms of $P$ and $Q$, we deduce that

$$
\left(1-\frac{P}{Q}\right)\left(1-\frac{Q}{P}\right)=\left(\frac{5}{P}-Q\right)\left(P-\frac{1}{Q}\right) .
$$

This is equivalent to (3.1) and hence we complete the proof.

Using the definitions of $h_{k, n}$ and $h_{k, n}^{\prime}$, we have the following:

Corollary 3.2. For every positive real number n, we have

$$
\sqrt{5}\left(h_{5, n} h_{5, n / 4}^{\prime}+\frac{1}{h_{5, n} h_{5, n / 4}^{\prime}}\right)=\frac{h_{5, n}}{h_{5, n / 4}^{\prime}}+\frac{h_{5, n / 4}^{\prime}}{h_{5, n}}+4 .
$$


Proof. Letting $q=e^{-\pi \sqrt{n / 5}}$ in (1.3) and (1.4), we find that $P=5^{1 / 4} h_{5, n}$ and $Q=5^{1 / 4} h_{5, n / 4}^{\prime}$ in Theorem 3.1. Rewriting (3.1) in terms of $h_{5, n}$ and $h_{5, n / 4}^{\prime}$, we complete the proof.

Theorem 3.3. If $P=\frac{\varphi(q)}{\varphi\left(q^{5}\right)}$ and $Q=\frac{\varphi\left(-q^{2}\right)}{\varphi\left(-q^{10}\right)}$, then

$$
\left(\frac{P}{Q}\right)^{2}+\left(\frac{Q}{P}\right)^{2}+4=Q^{2}+\frac{5}{Q^{2}} \text {. }
$$

Proof. By Lemma 2.1(i) and (iii),

where $\beta$ has degree 5 over $\alpha$. Thus

$$
P=\sqrt{\frac{z_{1}}{z_{5}}} \text { and } Q=\sqrt{\frac{z_{1}}{z_{5}}}\left(\frac{1-\alpha}{1-\beta}\right)^{1 / 8},
$$

$$
\frac{Q^{2}}{P^{2}}=\left(\frac{1-\alpha}{1-\beta}\right)^{1 / 4}
$$

By Lemma 2.3,

$$
\left(\frac{\beta}{\alpha}\right)^{1 / 4}+\frac{P^{2}}{Q^{2}}-\left(\frac{\beta}{\alpha}\right)^{1 / 4} \frac{P^{2}}{Q^{2}}=P^{2}
$$

and

$$
\left(\frac{\alpha}{\beta}\right)^{1 / 4}+\frac{Q^{2}}{P^{2}}-\left(\frac{\alpha}{\beta}\right)^{1 / 4} \frac{Q^{2}}{P^{2}}=\frac{5}{P^{2}} .
$$

Combining and rewriting above two equations in terms of $P$ and $Q$ as in the proof of Theorem 3.1, we complete the proof.

Using the definitions of $h_{k, n}$ and $h_{k, n}^{\prime}$, we have the following:

Corollary 3.4. For every positive real number $n$, we have

$$
\sqrt{5}\left(h_{5, n}^{\prime 2}+\frac{1}{h^{\prime 2}{ }_{5, n}}\right)=\left(\frac{h_{5, n}}{h_{5, n}^{\prime}}\right)^{2}+\left(\frac{h_{5, n}^{\prime}}{h_{5, n}}\right)^{2}+4 .
$$

Proof. Letting $q=e^{-\pi \sqrt{n / 5}}$ in (1.3) and (1.4), we find that $P=5^{1 / 4} h_{5, n}$ and $Q=5^{1 / 4} h_{5, n}^{\prime}$ in Theorem 3.3. Rewriting (3.3) in terms of $h_{5, n}$ and $h_{5, n}^{\prime}$, we complete the proof.

Theorem 3.5. If $P=\frac{\varphi(q)}{\varphi\left(q^{5}\right)}$ and $Q=\frac{\psi(q)}{q^{1 / 2} \psi\left(q^{5}\right)}$, then

$$
\left(\frac{P}{Q}\right)^{2}+\left(\frac{Q}{P}\right)^{2}+4=Q^{2}+\frac{5}{Q^{2}} \text {. }
$$

Proof. By Lemmas 2.1(i) and 2.2(i),

$$
P=\sqrt{\frac{z_{1}}{z_{5}}} \text { and } Q=\sqrt{\frac{z_{1}}{z_{5}}}\left(\frac{\alpha}{\beta}\right)^{1 / 8},
$$


where $\beta$ has degree 5 over $\alpha$. Thus

$$
\frac{Q^{2}}{P^{2}}=\left(\frac{\alpha}{\beta}\right)^{1 / 4}
$$

By Lemma 2.3,

$$
\frac{P^{2}}{Q^{2}}+\left(\frac{1-\beta}{1-\alpha}\right)^{1 / 4}-\frac{P^{2}}{Q^{2}}\left(\frac{1-\beta}{1-\alpha}\right)^{1 / 4}=P^{2}
$$

and

$$
\frac{Q^{2}}{P^{2}}+\left(\frac{1-\alpha}{1-\beta}\right)^{1 / 4}-\frac{Q^{2}}{P^{2}}\left(\frac{1-\alpha}{1-\beta}\right)^{1 / 4}=\frac{5}{P^{2}} .
$$

Combining and rewriting above two equations in terms of $P$ and $Q$ as in the proof of Theorem 3.1, we complete the proof.

Using the definitions of $h_{k, n}$ and $l_{k, n}^{\prime}$, we have the following:

Corollary 3.6. For every positive real number $n$, we have

$$
\sqrt{5}\left(l_{5, n}^{\prime 2}+\frac{1}{l^{\prime 2}{ }_{5, n}}\right)=\left(\frac{h_{5, n}}{l_{5, n}^{\prime}}\right)^{2}+\left(\frac{l_{5, n}^{\prime}}{h_{5, n}}\right)^{2}+4 .
$$

Proof. Letting $q=e^{-\pi \sqrt{n / 5}}$ in (1.3) and (1.6), we find that $P=5^{1 / 4} h_{5, n}$ and $Q=5^{1 / 4} l_{5, n}^{\prime}$ in Theorem 3.5. Rewriting (3.5) in terms of $h_{5, n}$ and $l_{5, n}^{\prime}$, we complete the proof.

Theorem 3.7. If $P=\frac{\psi(q)}{q^{1 / 2} \psi\left(q^{5}\right)}$ and $Q=\frac{\psi\left(q^{2}\right)}{q \psi\left(q^{10}\right)}$, then

$$
\left(\frac{P}{Q}\right)^{2}+\left(\frac{Q}{P}\right)^{2}+4=P^{2}+\frac{5}{P^{2}} \text {. }
$$

Proof. By Lemma 2.2(i) and (iii),

$$
P=\sqrt{\frac{z_{1}}{z_{5}}}\left(\frac{\alpha}{\beta}\right)^{1 / 8} \text { and } Q=\sqrt{\frac{z_{1}}{z_{5}}}\left(\frac{\alpha}{\beta}\right)^{1 / 4},
$$

where $\beta$ has degree 5 over $\alpha$. Thus

$$
\frac{Q^{2}}{P^{2}}=\left(\frac{\alpha}{\beta}\right)^{1 / 4} \quad \text { and } \quad \frac{z_{1}}{z_{5}}=\frac{P^{4}}{Q^{2}} .
$$

By Lemma 2.3,

$$
\frac{P^{2}}{Q^{2}}+\left(\frac{1-\beta}{1-\alpha}\right)^{1 / 4}-\frac{P^{2}}{Q^{2}}\left(\frac{1-\beta}{1-\alpha}\right)^{1 / 4}=\frac{P^{4}}{Q^{2}}
$$

and

$$
\frac{Q^{2}}{P^{2}}+\left(\frac{1-\alpha}{1-\beta}\right)^{1 / 4}-\frac{Q^{2}}{P^{2}}\left(\frac{1-\alpha}{1-\beta}\right)^{1 / 4}=\frac{5 Q^{2}}{P^{4}}
$$

Combining and rewriting above two equations in terms of $P$ and $Q$ as in the proof of Theorem 3.1, we complete the proof. 
Using the definition of $l_{k, n}^{\prime}$, we have the following:

Corollary 3.8. For every positive real number $n$, we have

$$
\sqrt{5}\left(l_{5, n}^{\prime 2}+\frac{1}{l^{\prime 2}{ }_{5, n}}\right)=\left(\frac{l_{5, n}^{\prime}}{l_{5,4 n}^{\prime}}\right)^{2}+\left(\frac{l_{5,4 n}^{\prime}}{l_{5, n}^{\prime}}\right)^{2}+4 .
$$

Proof. Letting $q=e^{-\pi \sqrt{n / 5}}$ in (1.6), we find that $P=5^{1 / 4} l_{5, n}^{\prime}$ and $Q=$ $5^{1 / 4} l_{5,4 n}^{\prime}$ in Theorem 3.7. Rewriting (3.7) in terms of $l_{5, n}^{\prime}$ and $l_{5,4 n}^{\prime}$, we complete the proof.

Theorem 3.9. If $P=\frac{\varphi\left(-q^{2}\right)}{\varphi\left(-q^{10}\right)}$ and $Q=\frac{\psi(q)}{q^{1 / 2} \psi\left(q^{5}\right)}$, then

$$
\left(P^{2}+Q^{2}-5\right)\left(\frac{1}{P^{2}}+\frac{1}{Q^{2}}-1\right)=1 .
$$

Proof. By Lemmas 2.1(iii) and 2.2(i),

$$
P=\sqrt{\frac{z_{1}}{z_{5}}}\left(\frac{1-\alpha}{1-\beta}\right)^{1 / 8} \text { and } Q=\sqrt{\frac{z_{1}}{z_{5}}}\left(\frac{\alpha}{\beta}\right)^{1 / 8}
$$

where $\beta$ has degree 5 over $\alpha$. Let $m=\frac{z_{1}}{z_{5}}$. Then

$$
\left(\frac{1-\alpha}{1-\beta}\right)^{1 / 4}=\frac{P^{2}}{m} \text { and }\left(\frac{\alpha}{\beta}\right)^{1 / 4}=\frac{Q^{2}}{m} .
$$

By Lemma 2.3, we deduce that

$$
\frac{1}{P^{2}}+\frac{1}{Q^{2}}-\frac{m}{P^{2} Q^{2}}=1
$$

and

$$
P^{2}+Q^{2}-\frac{P^{2} Q^{2}}{m}=5 .
$$

Combining and rewriting these two equations in terms of $P$ and $Q$, we complete the proof.

Using the definitions of $h_{k, n}^{\prime}$ and $l_{k, n}^{\prime}$, we have the following:

Corollary 3.10. For every positive real number $n$, we have

$$
\left(h_{5, n}^{\prime 2}+l_{5, n}^{\prime 2}-\sqrt{5}\right)\left(\frac{1}{h_{5, n}^{\prime 2}}+\frac{1}{l_{5, n}^{\prime 2}}-\sqrt{5}\right)=1 .
$$

Proof. Letting $q=e^{-\pi \sqrt{n / 5}}$ in (1.4) and (1.6), we find that $P=5^{1 / 4} h_{5, n}^{\prime}$ and $Q=5^{1 / 4} l_{5, n}^{\prime}$ in Theorem 3.9. Rewriting (3.9) in terms of $h_{5, n}^{\prime}$ and $l_{5, n}^{\prime}$, we complete the proof. 
Theorem 3.11. If $P=\frac{\psi(q)}{q^{1 / 2} \psi\left(q^{5}\right)}$ and $Q=\frac{\psi(-q)}{q^{1 / 2} \psi\left(-q^{5}\right)}$, then

$$
\left(P^{2}-Q^{2}-5\right)\left(\frac{1}{P^{2}}-\frac{1}{Q^{2}}-1\right)=1 \text {. }
$$

Proof. By Lemma 2.2(i) and (ii),

$$
P=\sqrt{\frac{z_{1}}{z_{5}}}\left(\frac{\alpha}{\beta}\right)^{1 / 8} \text { and } Q=\sqrt{\frac{z_{1}}{z_{5}}}\left(\frac{\alpha(1-\alpha)}{\beta(1-\beta)}\right)^{1 / 8},
$$

where $\beta$ has degree 5 over $\alpha$. Let

$$
m=\frac{z_{1}}{z_{5}} \quad \text { and } \quad T=\sqrt{\frac{z_{1}}{z_{5}}}\left(\frac{1-\alpha}{1-\beta}\right)^{1 / 4} .
$$

Then

$$
\left(\frac{\beta}{\alpha}\right)^{1 / 4}=\frac{m}{P^{2}}, \quad\left(\frac{1-\beta}{1-\alpha}\right)^{1 / 4}=\frac{\sqrt{m}}{T}, \quad \text { and } \quad\left(\frac{\beta(1-\beta)}{\alpha(1-\alpha)}\right)^{1 / 4}=\frac{m}{Q^{2}} .
$$

By Lemma 2.3, we deduce that

and

$$
\frac{m}{P^{2}}+\frac{\sqrt{m}}{T}-\frac{m}{Q^{2}}=m
$$

$$
\frac{P^{2}}{m}+\frac{T}{\sqrt{m}}-\frac{Q^{2}}{m}=\frac{5}{m}
$$

Combining and rewriting above two equations in terms of $P$ and $Q$, we complete the proof.

Using the definitions of $l_{k, n}$ and $l_{k, n}^{\prime}$, we have the following:

Corollary 3.12. For every positive real number $n$, we have

$$
\left(l_{5, n}^{\prime 2}-l_{5, n}^{2}-\sqrt{5}\right)\left(\frac{1}{l_{5, n}^{\prime 2}}-\frac{1}{l_{5, n}^{2}}-\sqrt{5}\right)=1
$$

Proof. Letting $q=e^{-\pi \sqrt{n / 5}}$ in (1.5) and (1.6), we find that $P=5^{1 / 4} l_{5, n}^{\prime}$ and $Q=5^{1 / 4} l_{5, n}$ in Theorem 3.11. Rewriting (3.11) in terms of $l_{5, n}$ and $l_{5, n}^{\prime}$, we complete the proof.

\section{Evaluations of $h_{5, n}, h_{5, n}^{\prime}, l_{5, n}$, and $l_{5, n}^{\prime}$}

In this section, we evaluate some numerical values of $h_{5, n}, h_{5, n}^{\prime}, l_{5, n}$, and $l_{5, n}^{\prime}$ by using the explicit relations and formulas established in Section 3.

The following results exhibit a general method for evaluating the values of $l_{5,4^{n}}^{\prime}$ for all positive integers $n$. We show the case when $n=1$ and $n=2$.

Theorem 4.1. We have

(i) $l_{5,1}^{\prime}=\sqrt{\frac{1+\sqrt{5}}{2}+\sqrt{\frac{1+\sqrt{5}}{2}}}$, 
(ii) $l_{5,4}^{\prime}=\frac{1+\sqrt{5}}{2}+\sqrt{\frac{1+\sqrt{5}}{2}}$,

(iii) $l_{5,16}^{\prime}=\sqrt{(3+2 \sqrt{5})((1+\sqrt{5}) a-1)+\frac{1}{2}(3+\sqrt{5})^{2} \sqrt{8 a-5+\sqrt{5}}}$, where

$$
a=\frac{1+\sqrt{5}}{2}+\sqrt{\frac{1+\sqrt{5}}{2}} .
$$

Proof. For (i), letting $n=1$ in (3.6) and using $h_{5,1}=1$ from Lemma 2.4, we find that

$$
l_{5,1}^{\prime 4}-(1+\sqrt{5}) l_{5,1}^{\prime 2}+1=0 .
$$

Solving for $l_{5,1}^{\prime}$ and using the fact that $l_{5,1}^{\prime}>1$, we complete the proof.

For (ii), letting $n=1$ in (3.8) and putting the value of $l_{5,1}^{\prime}$ from the previous result of (i), and using the fact that $l_{5,4}^{\prime}>1$, we deduce that

$$
(3+\sqrt{5}) l_{5,4}^{\prime 2}-11-5 \sqrt{5}-(6+2 \sqrt{5}) \sqrt{2+\sqrt{5}}=0 .
$$

Solving for $l_{5,4}^{\prime}$ and using the fact that $l_{5,4}^{\prime}>1$ again, we complete the proof.

For (iii), let $n=4$ in (3.8), then we deduce that

$$
l_{5,16}^{\prime 4}-\left(\sqrt{5} l_{5,4}^{\prime 2}-4 l_{5,4}^{\prime 2}+\sqrt{5}\right) l_{5,16}^{\prime 2}+l_{5,4}^{\prime 4}=0 .
$$

Putting the value of $l_{5,4}^{\prime}$ from the previous result of (ii), solving for $l_{5,16}^{\prime}$, and using the fact that $l_{5,16}^{\prime}>1$, we complete the proof.

See Theorems 3.3(v) and 4.10(xi) in [13] for alternative proofs for Theorem 4.1(i) and (ii), respectively. By repeating the same argument as in the proof of Theorem 4.1, we can find the values of $l_{5,4^{n}}^{\prime}$ for $n=3,4,5, \ldots$

Theorem 4.2. We have

$$
\begin{aligned}
& \text { (i) } l_{5,4}=\sqrt{\frac{2(3+\sqrt{5})(4 a-1+\sqrt{5})+(3+\sqrt{5})^{2} \sqrt{8 a-5+\sqrt{5}}}{2(1+\sqrt{5})(\sqrt{5} a-1)}}, \\
& \text { (ii) } l_{5,16}=\sqrt{\frac{\sqrt{5} b^{2}-6 b+\sqrt{5}+\sqrt{\left(\sqrt{5} b^{2}-2 b+\sqrt{5}\right)\left(\sqrt{5} b^{2}-6 b+\sqrt{5}\right)}}{2(\sqrt{5} b-1)}},
\end{aligned}
$$

where

$$
a=\frac{1+\sqrt{5}}{2}+\sqrt{\frac{1+\sqrt{5}}{2}}
$$

and

$$
b=(3+2 \sqrt{5})((1+\sqrt{5}) a-1)+\frac{1}{2}(3+\sqrt{5})^{2} \sqrt{8 a-5+\sqrt{5}} .
$$

Proof. For (i), let $n=4$ in (3.12), then we deduce that

$$
\left(\sqrt{5} l_{5,4}^{\prime 2}-1\right) l_{5,4}^{4}-\left(\sqrt{5} l_{5,4}^{\prime 4}-6 l_{5,4}^{\prime 2}+\sqrt{5}\right) l_{5,4}^{2}-l_{5,4}^{\prime 4}+\sqrt{5} l_{5,4}^{\prime 2}=0 .
$$


Putting the value of $l_{5,4}^{\prime}$ from Theorem 4.1(ii), solving for $l_{5,4}$, and using the fact that $l_{5,4}$ has a real value greater than 1 , we complete the proof.

For (ii), let $n=16$ in (3.12) and replace $l_{5,4}^{\prime}, l_{5,4}$ by $l_{5,16}^{\prime}, l_{5,16}$, respectively. Then the rest of the proof is similar to that of Part (i).

Theorem 4.3. We have

$$
h_{5,1}^{\prime}=\sqrt{\frac{1+\sqrt{5}}{2}-\sqrt{\frac{1+\sqrt{5}}{2}}} .
$$

Proof. Letting $n=1$ in (3.4) and using the fact that $h_{5,1}=1$ from Lemma 2.4, we find that

$$
(\sqrt{5}-1) h_{5,1}^{\prime 4}-4 h_{5,1}^{\prime 2}+\sqrt{5}-1=0 .
$$

Solving for $h_{5,1}^{\prime}$ and using the fact that $0<h_{5,1}^{\prime}<1$, we complete the proof.

See Theorem 4.16 in [11] for an alternative proof for Theorem 4.3.

Theorem 4.4. We have

(i) $h_{5,4}=\frac{2 \sqrt{2 a}}{(3+\sqrt{2}+\sqrt{5}+\sqrt{10})(a-\sqrt{5})}$,

(ii) $h_{5,16}=\sqrt{\frac{\sqrt{5} b^{2}-4 b+\sqrt{5}+\sqrt{\left(\sqrt{5} b^{2}-2 b+\sqrt{5}\right)\left(\sqrt{5} b^{2}-6 b+\sqrt{5}\right)}}{2}}$,

where

$$
a=\frac{1+\sqrt{5}}{2}+\sqrt{\frac{1+\sqrt{5}}{2}}
$$

and

$$
b=(3+2 \sqrt{5})((1+\sqrt{5}) a-1)+\frac{1}{2}(3+\sqrt{5})^{2} \sqrt{8 a-5+\sqrt{5}} .
$$

Proof. For (i), let $n=4$ in (3.2), then we find that

$$
\left(\sqrt{5} h_{5,1}^{\prime 2}-1\right) h_{5,4}^{2}-4 h_{5,1}^{\prime} h_{5,4}-h_{5,1}^{\prime 2}+\sqrt{5}=0 .
$$

Note that

$$
\frac{1+\sqrt{5}}{2}-\sqrt{\frac{1+\sqrt{5}}{2}}=\left(\frac{1+\sqrt{5}}{2}+\sqrt{\frac{1+\sqrt{5}}{2}}\right)^{-1} .
$$

Putting the value of $h_{5,1}^{\prime}$ from Theorem 4.3, solving for $h_{5,4}$, and using the fact that $h_{5,4}>0$, we complete the proof.

For (ii), let $n=16$ in (3.6), then we deduce that

$$
h_{5,16}^{4}-\left(\sqrt{5} l_{5,16}^{\prime 4}-4 l_{5,16}^{\prime 2}+\sqrt{5}\right) h_{5,16}^{2}+l_{5,16}^{\prime 4}=0 .
$$

Putting the value of $l_{5,16}^{\prime}$ from Theorem 4.1(iii), solving for $h_{5,16}$, and using the fact that $0<h_{5,16}<1$, we complete the proof. 


\section{Evaluations of $R(q)$ and $S(q)$}

We now apply specific values of $h_{5, n}$ and $l_{5, n}$ to evaluations of the RogersRamanujan continued fraction. In his second letter to G. H. Hardy (see [5] for more details), Ramanujan gave the first non-elementary evaluations of $R(q)$ and $S(q)$, namely,

and

$$
R\left(e^{-2 \pi}\right)=\sqrt{\frac{5+\sqrt{5}}{2}}-\frac{\sqrt{5}+1}{2}
$$

$$
S\left(e^{-\pi}\right)=\sqrt{\frac{5-\sqrt{5}}{2}}-\frac{\sqrt{5}-1}{2} .
$$

Berndt, Chan, and Zhang [4] derived formulas for the explicit evaluations of $R\left(e^{-2 \pi \sqrt{n}}\right)$ and $S\left(e^{-\pi \sqrt{n}}\right)$ for positive rational numbers $n$ in terms of Ramanujan-Weber class invariants. In [10], Yi established some formulas and determined values for $R\left(e^{-2 \pi \sqrt{n}}\right)$ and $S\left(e^{-\pi \sqrt{n}}\right)$ by using modular equations of degrees 5 or 25 . She provided simple proofs for some known values of $R(q)$ and also found some new values of $R(q)$. We now consider the numerical values of $R\left(e^{-2 \pi \sqrt{n / 5}}\right)$ and $S\left(e^{-\pi \sqrt{n / 5}}\right)$ where $n$ is a positive integer. In [9], the values of $R\left(e^{-2 \pi \sqrt{n / 5}}\right)$ were evaluated when $n=1,2,3,4,7,8,9$ and the values of $S\left(e^{-\pi \sqrt{n / 5}}\right)$ were evaluated when $n=1,3,7,9$. We end this section by evaluating the numerical values of $R\left(e^{-2 \pi \sqrt{n / 5}}\right)$ and $S\left(e^{-\pi \sqrt{n / 5}}\right)$ for $n=4$ and $n=16$.

\section{Theorem 5.1. We have}

(i) $R^{5}\left(e^{-4 \pi / \sqrt{5}}\right)=\frac{1}{2}\left(-11-5 \sqrt{5} b+\sqrt{5\left(25 b^{2}+22 \sqrt{5} b+25\right)}\right)$,

(ii) $S^{5}\left(e^{-2 \pi / \sqrt{5}}\right)=\frac{1}{2}\left(11-5 \sqrt{5} c+\sqrt{5\left(25 c^{2}-22 \sqrt{5} c+25\right)}\right)$,

where

$$
\begin{aligned}
& a=\frac{1+\sqrt{5}}{2}+\sqrt{\frac{1+\sqrt{5}}{2}} \\
& b=2 a\left(\frac{(3+\sqrt{5})(2(4 a+1-\sqrt{5})+(3+\sqrt{5}) \sqrt{8 a-5+\sqrt{5}})}{(1+\sqrt{5})(3+\sqrt{2}+\sqrt{5}+\sqrt{10})(\sqrt{5} a-1)(a-\sqrt{5})}\right)^{2} \\
& c=\frac{32(3+\sqrt{5}) a^{2}(2(4 a+1-\sqrt{5})+(3+\sqrt{5}) \sqrt{8 a-5+\sqrt{5}})}{(1+\sqrt{5})(\sqrt{5} a-1)((3+\sqrt{2}+\sqrt{5}+\sqrt{10})(a-\sqrt{5}))^{4}}
\end{aligned}
$$

Proof. The results follow directly form Lemma 2.6 and Theorems 4.2(i) and 4.4(i).

See [11] for an alternative proof of Theorem 5.1(i). 
Theorem 5.2. We have

(i) $R^{5}\left(e^{-8 \pi / \sqrt{5}}\right)=\frac{1}{2}\left(-11-5 \sqrt{5} c+\sqrt{5\left(25 c^{2}+22 \sqrt{5} c+25\right)}\right)$,
(ii) $S^{5}\left(e^{-4 \pi / \sqrt{5}}\right)=\frac{1}{2}\left(11-5 \sqrt{5} d+\sqrt{5\left(25 d^{2}-22 \sqrt{5} d+25\right)}\right)$

where

$$
\begin{aligned}
& a=\frac{1+\sqrt{5}}{2}+\sqrt{\frac{1+\sqrt{5}}{2}} \\
& b=(3+2 \sqrt{5})((1+\sqrt{5}) a-1)+\frac{1}{2}(3+\sqrt{5})^{2} \sqrt{8 a-5+\sqrt{5}} \\
& c=\frac{b^{2}\left(\sqrt{5} b^{2}-6 b+\sqrt{5}\right)}{(\sqrt{5} b-1)^{2}}, \\
& d=\frac{8 b^{4} \sqrt{\sqrt{5} b^{2}-6 b+\sqrt{5}}}{(\sqrt{5} b-1)\left(\sqrt{\sqrt{5} b^{2}-2 b+\sqrt{5}}+\sqrt{\sqrt{5} b^{2}-6 b+\sqrt{5}}\right)^{3}} .
\end{aligned}
$$

Proof. The results follow directly form Lemma 2.6 and Theorems 4.2(ii) and 4.4(ii).

\section{References}

[1] B. C. Berndt, Number Theory in the Spirit of Ramanujan, American Mathematical Society, 2006.

[2] — Ramanujan's Notebooks, Part III, Springer-Verlag, New York, 1991.

[3] _ Ramanujan's Notebooks, Part IV, Springer-Verlag, New York, 1991.

[4] B. C. Berndt, H. H. Chan, and L. C. Zhang, Explicit Evaluations of the RogersRamanujan continued fraction, J. Reine Angew. Math. 480 (1996), 141-159.

[5] B. C. Berndt and R. A. Rankin, Ramanujan: Essays and Surveys, American Mathematical Society, Providence, 2001; London Mathematical Society, London, 2001.

[6] D. H. Paek and J. Yi, On some modular equations and their applications II, Bull. Korean Math. Soc. 50 (2013), no. 4, 1221-1233.

[7] R. A. Rankin, Modular Forms and Functions, Cambridge University Press, Cambridge, 1977.

[8] B. Schoeneberg, Elliptic Modular Functions, Springer-Verlag, New York, 1974.

[9] J. Yi, The Construction and Applications of Modular Equations, Ph. D. Thesis, University of Illinois at Urbana-Champaign, 2001.

[10] - Evaluations of the Rogers-Ramanujan continued fraction $R(q)$ by modular equations, Acta Arith. 97 (2001), no. 2, 103-127.

[11] _ Theta-function identities and the explicit formulas for theta-function and their applications, J. Math. Anal. Appl. 292 (2004), no. 2, 381-400.

[12] J. Yi, M. G. Cho, J. H. Kim, S. H. Lee, J. M. Yu, and D. H. Paek On some modular equations and their applications I, Bull. Korean Math. Soc. 50 (2013), no. 3, 761-776.

[13] J. Yi, Y. Lee, and D. H. Paek, The explicit formulas and evaluations of Ramanujan's theta-function $\psi$, J. Math. Anal. Appl. 321 (2006), no. 1, 157-181. 
Dae Hyun Paek

Department of Mathematics Education

Busan National University of Education

BUSAN 611-736, Korea

E-mail address: paek@bnue.ac.kr

JINHEE YI

Department of Mathematics and Computer Science

Korea Science Academy of KAIST

Busan 614-822, Korea

E-mail address: jhyi100@kaist.ac.kr 